\title{
Alterstice
}

Revue internationale de la recherche interculturelle

International Journal of Intercultural Research

Revista International de la Investigacion Intercultural

\section{Les représentations filmiques de la santé mentale des migrants : pistes réflexives}

\section{Mouloud Boukala}

Volume 4, numéro 2, 2014

URI : https://id.erudit.org/iderudit/1077428ar

DOI : https://doi.org/10.7202/1077428ar

Aller au sommaire du numéro

Éditeur(s)

Alterstice

ISSN

1923-919X (numérique)

Découvrir la revue

Citer cet article

Boukala, M. (2014). Les représentations filmiques de la santé mentale des migrants : pistes réflexives. Alterstice, 4(2), 85-98.

https://doi.org/10.7202/1077428ar

\section{Résumé de l'article}

Cet article propose des pistes réflexives sur l'analyse des représentations cinématographiques, principalement fictionnelles, abordant la santé mentale des migrants. L'un des enjeux de notre réflexion est de cerner les films se consacrant à ce sujet et, de les identifier puis d'analyser comment des individus - en l'occurrence des migrants malades - y sont représentés. Qu'apporte ce mode de figuration qu'est le cinéma à la compréhension de la santé mentale en contexte migratoire ? Le cinéma fictionnel est-il source de stéréotypie (les migrants comme porteurs de danger social, responsables des déficits publics, etc.) ou marque-t-il une rupture avec des représentations réductrices ? Le cinéma met-il en image et en sons les impacts des violences, de l'isolement affectif, de la guerre, des persécutions, des menaces, des troubles du sommeil sur la santé mentale des migrants ? La fiction rend-elle compte des espaces cliniques et thérapeutiques proposés aux migrants ? 


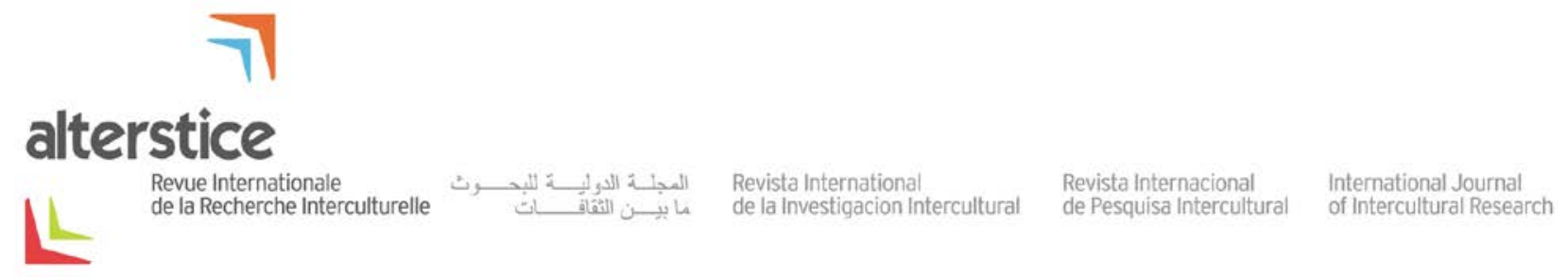

ARTICLE HORS THÈME

Les représentations filmiques de la santé mentale des migrants : pistes réflexives

Mouloud Boukala ${ }^{1}$

\section{Résumé}

Cet article propose des pistes réflexives sur l'analyse des représentations cinématographiques, principalement fictionnelles, abordant la santé mentale des migrants. L'un des enjeux de notre réflexion est de cerner les films se consacrant à ce sujet et, de les identifier puis d'analyser comment des individus - en l'occurrence des migrants malades - y sont représentés. Qu'apporte ce mode de figuration qu'est le cinéma à la compréhension de la santé mentale en contexte migratoire ? Le cinéma fictionnel est-il source de stéréotypie (les migrants comme porteurs de danger social, responsables des déficits publics, etc.) ou marque-t-il une rupture avec des représentations réductrices ? Le cinéma met-il en image et en sons les impacts des violences, de l'isolement affectif, de la guerre, des persécutions, des menaces, des troubles du sommeil sur la santé mentale des migrants? La fiction rend-elle compte des espaces cliniques et thérapeutiques proposés aux migrants?

\section{Rattachement de l'auteur}

${ }^{1}$ Université du Québec à Montréal, Montréal

\section{Correspondance}

boukala.mouloud@uqam.ca

\section{Mots clés}

migrations; santé mentale; cinéma; fictions; esclavage; guerre.

\section{Pour citer cet article}

Boukala, M. (2014). Les représentations filmiques de la santé mentale des migrants : pistes réflexives. Alterstice, $4(2), 85-98$. 
Cet article propose des pistes réflexives sur l'analyse des représentations cinématographiques, principalement fictionnelles ${ }^{1}$, abordant la santé mentale des migrants ${ }^{2}$. L'un des enjeux de notre réflexion est de cerner les films se consacrant à ce sujet et de les identifier puis d'analyser comment des individus - en l'occurrence des migrants malades - y sont représentés. Qu'apporte ce mode de figuration qu'est le cinéma à la compréhension de la santé mentale en contexte migratoire? Le cinéma fictionnel est-il source de stéréotypie (les migrants comme porteurs de danger social, responsables des déficits publics, etc.) ou marque-t-il une rupture avec des représentations réductrices? Le cinéma met-il en images et en sons les impacts des violences, de l'isolement affectif, de la guerre, des persécutions, des menaces, des troubles du sommeil sur la santé mentale des migrants? La fiction rend-elle compte des espaces cliniques et thérapeutiques proposés aux migrants?

Autant d'interrogations qui feront la matière de cet article où le cinéma ne sera pas envisagé comme une illustration d'une situation sociale (celle du migrant) et d'un état (sa santé mentale) mais considéré comme un mode de connaissance susceptible de rendre compte des conséquences de processus migratoires sur la santé de personnes déplacées. Dans la perspective anthropologique qui est ici la nôtre, nous envisagerons comment le cinéma fictionnel s'avère riche de potentialités en révélant la manière dont des difficultés prémigratoires et post migratoires génèrent des situations problématiques et vulnérabilisantes, affectant la santé mentale des migrants. Chaque film sera considéré comme " un produit social déterminé » (Morin, 1956, p. 196) susceptible de rendre compte de l' « esprit du temps " d’une société (Morin, 2009) et de " comment parler de la société » (Becker, 2009). Le cinéma narratif ne serait être l'expression transparente de la réalité sociale ou son reflet (Sorlin, 1972 et 1977), mais sera plutôt appréhendé comme une occasion d'enrichissement humain ${ }^{3}$ et de " transferts imaginaires ${ }^{4}$ " pour reprendre une expression de Morin.

Cet article comprend quatre moments réflexifs. Le premier moment vise à rendre compte de la manière dont l'immigration et la maladie mentale peuvent être envisagées comme des expériences-limites au sens de Foucault (Calderon, 2003). Dans un deuxième temps, l'accent sera mis sur les nombreuses représentations filmiques de la santé mentale de personnes non migrantes. Nous poursuivrons dans une troisième partie par une réflexion sur les divers aspects de l'expérience migratoire (le départ, le trajet, le contrôle aux frontières, l'arrivée, l'acculturation, etc.) dans les films fictionnels, avant d'envisager les représentations de la santé mentale des migrants dans le cinéma de fiction. Enfin, dans une dernière partie, une incursion dans le cinéma documentaire contemporain d'animation permettra de présenter des outils filmiques pédagogiques ayant pour fonction de sensibiliser à la compréhension des problèmes des migrants (difficultés communicationnelles liées à la barrière de la langue, souvenirs douloureux des interrogatoires lorsque le migrant se trouve dans le bureau du médecin, etc.) et de proposer certaines pistes de réflexion en vue de meilleures pratiques professionnelles.

\section{L'immigration et la maladie mentale comme expériences limites}

La maladie mentale et la condition des migrants présentent de nombreuses caractéristiques communes ${ }^{5}$. Les deux constituent ce que Michel Foucault nommait des expériences limites, " ces formes d'expérience qui au lieu d'être considérées comme centrales, d'être valorisées positivement dans une société, sont considérées comme des expériences limites, comme des expériences frontières, à partir de quoi est remis en question cela même qui est considéré comme d’ordinaire acceptable " (cité dans Calderon, 2003). Ces expériences limites sont toutes deux,

\footnotetext{
${ }^{1}$ Seule la dernière partie de l'article est consacrée au cinéma documentaire. Cette brève incursion dans le cinéma documentaire d'animation à visée pédagogique se trouvera justifiée au fur et à mesure des étapes de notre réflexion.

${ }^{2}$ Nous tenons à remercier chaleureusement Joseph Lévy qui, grâce à ses relectures critiques, a enrichi notre réflexion, ainsi que Rachel Trahan Brousseau et Jason Burnham qui m’ont permis de prendre connaissance de films en lien avec la thématique abordée.

${ }^{3}$ Morin a cette phrase très à propos dans le cadre de cet article: " Le spectateur de cinéma sympathise avec le vagabond Charlot, alors que dans la vie réelle il se détourne quand un vagabond lui demande un peu d'argent. Nous sommes ainsi beaucoup plus humains et plus intelligents au cinéma parce que nous comprenons autrui alors que dans la vie réelle nous nous fermons à cette compréhension d'autrui » (2009, p. 20).

${ }^{4}$ Morin précise que : "l'imaginaire est le ferment du travail de soi sur soi et sur la nature à travers lequel se construit et se développe la réalité de l'homme " (1956, p. 212).

${ }^{5}$ Certains auteurs, à l'instar de Susan Sontag, considèrent que « la folie constitue une forme d'exil » (2005, p. 50).
} 
sous diverses formes, tenues à distance du reste de la population par diverses mesures institutionnelles et gouvernementales. En témoignent l'apparition de murs et la militarisation de la " police des frontières » qui entravent et nient toute diversité. Cette mise à distance " intelligente ", basée notamment sur l'achat de drones et d'outils de détection, a pour but de diminuer l'immigration clandestine aux États-Unis. Ce faisant, elle transforme le migrant en un étranger anthropologique dans un territoire frontalier, comme l'explique Michel Agier :

L'extraterritorialité est l'illusion première de cet étranger-là : ce qui fonde son étrangeté "radicale" est une altérité biopolitique, produite par le gouvernement "technique" d'une catégorie de population à part. Cette altérité radicale imposée par l'instauration de murs et de barrières plus difficiles à franchir est, en termes relationnels, première par rapport à une différence culturelle qui n'a pas de lieu où être mise à l'épreuve. (2013, p. 87)

Si l'histoire de la folie offre à Foucault l'occasion de s'interroger sur le système de raison occidentale, les préalables sur la migration et la santé mentale nous amènent :

- à amorcer une réflexion sur les migrants, c'est-à-dire des présences déplacées dans le temps et l'espace et neutralisées politiquement ${ }^{6}$

- à considérer le migrant comme un « hybride culturel qui vit à la frontière de deux modèles différents de vie, sans savoir auquel des deux il appartient » (Schütz, 2003, p. 91). Les contradictions d'un double référent sont susceptibles de générer des confits et des crises dans la mesure où « ils [les immigrants] ne savaient plus à quel environnement culturel et symbolique ils étaient le plus attachés »(Ben Jelloun, 1977, p. 21),

- à envisager le migrant comme un " composé spécial de proximité et d’éloignement, d’indifférence et d'engagement " ${ }^{7}$ (Simmel, 2010, p. 665) qui oscille " sans cesse entre la réserve et l'intimité » (Schütz, 2003, p. 35), doté de «la conscience de n'avoir en commun que le simple universel [qui] met davantage en relief justement ce qui n'est pas commun » (Simmel, 2010, p. 667). À l'instar de la vision romantique de la maladie (Sontag, 2005, p. 50), la migration exacerbe la conscience. Cette condition d'exilé suscite cette " conscience contrapuntique ", ce décentrement, cette conscience non continue de plusieurs cultures et de plusieurs mondes ${ }^{8}$ et cette objectivité qui « lui a enseigné qu'un homme peut toujours perdre son statut, ses règles de vie et même sa propre histoire » (Schütz, 2003, p. 36)

- à déconstruire certaines représentations romanesques de l’immigration véhiculées par le cinéma et la littérature comme celle d'une « immigration de peuplement, d'une immigration volontariste et conquérante, sereinement détachée de toute idée de retour » (Sayad, 2006, p. 169)

- à interroger la médiation institutionnelle proposée ou imposée au migrant et apprécier si elle favorise le passage de l' " esquisse d'homme " à "son épanouissement humain ", la transition de " l'homme capable au citoyen réel » (Ricœur, 1995, p. 39-40)

- à relever les stratégies identitaires ${ }^{9}$ déployées par les individus et à envisager la maladie et la victimisation comme des stratégies identitaires possibles (Benbassa, 2007; Fassin et Rechtman, 2011; Vasquez, 1990). L'hospitalisation peut être envisagée comme une stratégie identitaire et s'accompagner d'une forme de reconnaissance dans la mesure où le migrant est reconnu comme un malade à qui l'on prodigue des soins et qui fait l'objet d'attention

\footnotetext{
${ }^{6}$ « provisoire en droit n'ayant pas sa fin en elle-même, présence déplacée, présence extra-ordinaire, la présence immigrée est tenue, ainsi que le veut et l'exige la logique de l'Etat, de se conformer à la neutralité politique [...] l'immigration est neutralisée politiquement » (Sayad, 2006, p. 165).

${ }^{7}$ Schütz précise en d'autres mots que « ce qui fait défaut à l'étranger, c'est qu'il ne sait pas instinctivement trouver la bonne distance avec les autres » (2003, p. 35).

8 "L'exil est nomade, décentré, contrapuntique et, dès que l'on s'y habitue, sa force déstabilisante surgit à nouveau » (Saïd, 2008, p. 257).

9 Par stratégies identitaires, nous nous référons à des « procédures mises en œuvre (de façon consciente ou inconsciente) par un acteur (individuel ou collectif) pour atteindre une, ou des, finalités (définies explicitement ou se situant au niveau de l'inconscient), procédures élaborées en fonction de la situation d'interaction, c'est-à-dire en fonction des différentes déterminations (socio-historiques, culturelles, psychologiques) de cette situation » (Lipiansky, Taboada-Leonetti et Vasquez, 1990, p. 24).
} 
- confronter la visibilité sociale ${ }^{10}$ des migrants (leur reconnaissance par leur prise en compte notamment grâce à l'hospitalisation) et leur visibilité (ou traitement) médiatique.

Si ces pistes réflexives seront développées dans des recherches ultérieures par des analyses de cas, elles nous permettent déjà de porter une attention plus précise sur la représentation de la santé mentale au cinéma, puis sur celle du migrant au grand écran.

\section{Remarques sur les liens entre cinéma fictionnel et santé mentale}

Dès les années 1950, des cinéastes (Mankiewicz, Franju, Hitchcock, Polanski, etc.) s'intéressent à la maladie mentale et $n^{\prime}$ hésitent pas à la porter à l'écran ${ }^{11}$. Cet intérêt marqué de leur part se poursuit dans les décennies subséquentes (Fuller, Antonioni, Cassavetes, Truffaut, Forman, Loach, Beineix, etc.) et ne se dément pas, comme en attestent des réalisations récentes (Shutter Island, Scorsese, 2010; Black Swan, Aronofksy, 2010; A Dangerous Method, Cronenberg, 2011; Jimmy P., Desplechin, 2013). Trois éléments, entre autres, sont susceptibles d'expliquer cet intérêt de la fiction pour la santé mentale : la dimension spectaculaire que ces films permettent, la dimension identificatoire qu'ils autorisent et enfin les rebondissements scénaristiques qu'ils offrent.

La dimension spectaculaire est un aspect récurrent des films traitant de santé mentale (Gordon, 2013; Zarifian, 2006). Michel Foucault n'a jamais été aussi près de définir le regard cinématographique que lorsqu'il écrit : « Le regard clinique a cette paradoxale propriété d'entendre un langage au moment où il perçoit un spectacle (2005, p. 108) [...] Un regard qui écoute et un regard qui parle : l'expérience clinique représente un moment d'équilibre entre la parole et le spectacle » (p. 116). L'adjectif spectaculaire est défini ainsi dans Le Trésor de la langue française : " qui frappe la vue, l'imagination par son caractère remarquable, les émotions, les réflexions suscitées " (Quemada, 1992, p. 857). Des films comme Shock Corridor (Fuller, 1963) A Woman Under the Influence (Cassavetes, 1974), One Flew Over the Cuckoo's Nest (Forman, 1975) et A Beautiful Mind (Howard, 2001) sont spectaculaires par la démesure de ce qui nous est présenté visuellement et auditivement (crises et traitements thérapeutiques, notamment ceux par électrochocs), les effets spéciaux liés à ce spectacle (délires, hallucinations, dédoublements de la personnalité) et l'emphase mise sur les performances des acteurs (Peter Breck, Jack Nicholson ${ }^{12}$, Russell Crowe), et de l'actrice (Gena Rowlands). Ces moments spectaculaires, c'est-à-dire " propres à constituer un spectacle », amplifient une action et la font progresser. À titre d'exemple, dans Shock Corridor (Fuller, 1963), Johnny Barrett (Peter Breck), journaliste arriviste du Daily Globe, décide de mener une enquête sur un meurtre commis dans une " clinique mentale ». Le seul moyen de s'introduire dans l'institution afin d'écrire l'article qui, selon lui, le mènera au Pulitzer, revient à simuler la folie. Il dresse, compose et peaufine le portrait d'un " obsédé sexuel ». Face aux spécialistes de l'institution, il s'agite, il hurle, il exige qu'on reconnaisse sa bonne santé mentale. Et lors des entretiens, il passe violemment à l'acte en agressant physiquement le médecin. Son comportement spectaculaire choque et persuade. Le diagnostic est sans appel. Johnny Barrett est interné. De plus, ces moments spectaculaires "frappent l'imagination » et produisent des effets immédiats sur le spectateur en devenant des catalyseurs de l'émotion, car comme l'observe André Gardies : "le spectaculaire n'est pas d'ordre essentiel, mais de l'ordre de l'effet. C'est des conditions d'émergence et d'organisation de l'événement que naît l'effet spectaculaire, plus précisément ce sont elles qui font que l'événement est reçu comme spectaculaire » (Hamon-Sirejols et Gardies, 1997, p. 120).

Des films traitant de santé mentale (Family Life, Loach, 1971; Birdy, Parker, 1984; The Mosquito Coast, Weir, 1986; $\mathrm{Pi}$, Aronofsky, 1998) suscitent chez le spectateur une inquiétante étrangeté: « L'inquiétante étrangeté qui s'attache à l'épilepsie, à la folie a la même origine. Le profane se voit là confronté à la manifestation de forces qu'il ne présumait pas chez son semblable, mais dont il lui est donné de ressentir obscurément le mouvement dans des coins reculés de sa propre personnalité ॥ (Freud, 1985, p. 249). Il est possible d'émettre l'hypothèse selon laquelle

\footnotetext{
${ }^{10}$ Comme le rappelle Serge Moscovici, "Quel que soit le sacrifice, leur premier souci est en fait de devenir visibles, donc d'obtenir la pleine reconnaissance de leur existence aux yeux de la majorité et dans l'esprit de ceux qui la composent » (1991, p. 224).

${ }^{11}$ Cet article ne vise pas à présenter un corpus filmique exhaustif. Ainsi, d'autres films abordant ce sujet sont antérieurs aux années 1950 : The Three Faces of Eve, Johnson, 1957; Spellbound, Hitchcock, 1948; etc.

12 Oscar du Meilleur acteur en 1976.
} 
ces films libèrent notre mauvaise conscience car «le fou constitue - comme le suggère Roger Bastide - une expression de notre "mauvaise conscience", le double extériorisé et agissant en public, de cette part de chaos que nous voulons nier en nous-mêmes. C'est pourquoi nous le considérons comme un "hors-la-loi", comme un danger pour la société; les jugements que nous portons sur lui ne sont que le reflet ou, mieux, la projection du jugement que nous portons sur une part de nous-mêmes » (1965, p. 307).

Enfin, la maladie mentale offre de nombreux rebondissements aux scénaristes qui créent des relations jusqu'alors inédites sous forme de rencontres en rapprochant au sein d'espaces cliniques des personnages qui ne seraient pas amenés à se croiser dans leur société (Shock Corridor, Fuller, 1963; One Flew Over the Cuckoo's Nest, Forman, 1975; Shutter Island, Scorsese, 2010) ou alors en éloignant des personnages proches au sein d'une famille (Family Life, Loach, 1971; Rain Man, Levinson, 1988). La santé mentale au cinéma, semblable en cela à une table de montage, réunit ceux (malades et bien-portants) que généralement leur société sépare. Elle permet dans bien des cas de pénétrer dans des huis clos anthropologiques et de découvrir des « hommes d’un autre monde » (Ey, 1952, p. 892). Prenons par exemple un film réunissant, au sein d'un espace clinique, des protagonistes qui ne se connaissent pas, qui ne pas seraient pas croisés dans leur société et qui pourtant seront amenés à vivre ensemble. Dans One Flew Over the Cuckoo's Nest (Forman, 1975), nous assistons à l'arrivée menottée de Randle Patrick McMurphy (Jack Nicholson) dans un hôpital psychiatrique. Il est transféré de la Ferme d'État de Pendelton où on le soupçonne de simuler la folie afin d'éviter toute forme de travail. Le film repose sur la rencontre et les interactions entre le protagoniste principal et l'infirmière en chef d'une part, et celles avec les autres patients (Harding, Cheswick, Scanlon, Billy, Martini, Bancini, Chief Bromden), qui pour certains résident dans l'établissement psychiatrique de leur plein gré.

Signalons en nous inspirant des travaux de Talcott Parsons (1955) que l'individu malade au cinéma est susceptible de transgresser les normes sociales et les valeurs d'une société (et de porter atteinte à sa structure et à son fonctionnement) à quatre niveaux différents ${ }^{13}$ :

a) celui de la famille ou du couple (La tête contre les murs, Franju, 1959; Suddenly, Last Summer, Mankiewicz, 1959; Family Life, Loach, 1971; A Woman Under the Influence, Cassavetes, 1974; Salto nel vuoto [Le saut dans le vide], Bellocchio, 1980; $37^{\circ} 2$ le matin, Beineix, 1985; Rain Man, Levinson, 1988; Shine, Hicks, 1996),

b) celui du travail (The Mosquito Coast, Weir, 1986; An Angel at My Table, Campion, 1990; Pi, Aronofsky, 1998; A Beautiful Mind, Howard, 2001; A Dangerous Method, Cronenberg, 2011),

c) celui de la communauté (II deserto rosso [Le désert rouge], Antonioni, 1964; L'histoire d'Adèle H., Truffaut, 1975; One Flew Over the Cuckoo's Nest, Forman, 1975; Birdy, Parker, 1984; Mr. Jones, Figgis, 1993),

d) et celui de la société globale (Shock Corridor, Fuller, 1963; The Madness of King George, Hytner, 1994; Fight Club, Fincher, 1999 ; Girl, Interrupted, Mangold, 1999).

Au sortir de la vision de nombre de ces films (Suddenly, Last Summer, Mankiewicz, 1959; Shock Corridor, Fuller, 1963; One Flew Over the Cuckoo's Nest, Forman, 1975; Fight Club, Fincher, 1999; Rain Man, Levinson, 1988; Shutter Island, Scorsese, 2010) transparaissent trois aspects saillants et heuristiques qui seront par la suite utiles à l'étude des représentations filmiques de la santé mentale des migrants :

1) les protagonistes principaux sont majoritairement des non-migrants et représentent dans certains cas la diversité (ethnique, religieuse, politique) de manière indirecte. Dans Shock Corridor, trois patients ont assisté au meurtre commis dans la clinique: Stuart (sudiste, élevé par ses parents dans la haine des Yankees), Trent (le seul étudiant noir d'une université du Sud), Boden (physicien américain, prix Nobel de physique, spécialiste de la bombe atomique dont l'âge mental actuel est de six ans). Ainsi, c'est en se faisant passer pour un général sudiste, Nathan Bedford Forrest, en animant une émeute raciale au côté de Trent et en jouant comme un enfant de six ans que le journaliste glane les informations nécessaires à

\footnotetext{
13 Précisons que ces divers niveaux peuvent se recouper.
} 
l'identification du meurtrier. Il est également possible de voir en Randle Patrick McMurphy (One Flew Over the Cuckoo's Nest) l'Indien, le Sauvage qui tente d'insuffler une révolte au sein d'une institution (patrie) incarnée par l'infirmière, Miss Ratched.

2) le spectateur saisit à quel point existent au cinéma, des "façons correctes d'être fou ", ce que Ralph Linton appelle des "modèles d'inconduite » (1968). Le fou est souvent présenté comme intenable, imprévisible, dangereux (Shock Corridor). Il pousse des cris, déchire ses vêtements et détruit tout ce qui l'entoure. Il s'agite, parle sans suite ou s'enfonce dans un mutisme (One Flew Over the Cuckoo's Nest). Les films cités ci-dessus fournissent, dans bien des cas, des indications sur les " modes d'emploi abusif » et promeuvent des « valeurs sociales antisociales » (Devereux, 1977, p. 3) qui permettent à l'individu d'être antisocial d'une manière socialement approuvée. Ce sont ces directives explicites que Linton désigne par " modèles d'inconduite » et qu'il résume par cette adresse au " déviant ": " Ne faites pas ceci, mais si vous le faites, il faut vous y prendre de cette façon » (p. 461-462). De ce qui précède, il résulte que les gestes ne servent pas uniquement à produire une action mais également à signifier à autrui une situation et un état. On n'est fou que dans et par rapport à une société donnée et selon des modalités qui sont culturellement façonnées.

3) Ces productions privilégient en grande partie le recours au spectaculaire (le jeu performant) et attachent moins d'importance à une dimension plus complexe, celle de la souffrance du malade (le " je » souffrant). Pour certaines d'entre elles, nous sommes loin du commentaire de Jean-Paul Sartre suscité à la vision du documentaire Regard sur la folie (Ruspoli, 1962) : "il [ce film] nous invite par d'admirables images à faire pour la première fois l'expérience de la maladie mentale; par tout ce qu'elle a de si proche et de si lointain, elle nous fait comprendre à la fois que tous les hommes ne sont pas fous mais que tous les fous sont des hommes » (Bovier, 2011).

Ces trois traits saillants ne manquent pas de soulever des interrogations quant aux éventuelles représentations filmiques de la santé mentale des migrants : l'expression de la santé mentale des migrants est-elle spectaculaire? Le je-souffrant est-il privilégié au je-performant? Les cinéastes donnent-ils à voir les politiques médicales de la société d'accueil envers les migrants?

Avant de revenir sur ces diverses interrogations et de soulever la problématique de la santé mentale dans des œuvres filmiques fictionnelles, nous décrirons les modalités avec lesquelles on porte les migrants au grand écran.

\section{Réflexions sur les liens entre cinéma fictionnel et immigration}

Les migrations et les immigrations sont des sujets qui ont été portées de nombreuses fois au grand écran ${ }^{14}$. Elles continuent encore aujourd'hui d'inspirer des auteurs qui œuvrent aussi bien dans le champ de la fiction que dans celui du cinéma documentaire. II y a pléthore de films retraçant les trajectoires individuelles et collectives de migrants, ces « personnes déplacées dans l’espace et le temps, et neutralisées politiquement ».

Au sein de fictions, les cinéastes abordent divers aspects que nous présenterons sous la forme d'une séquence chronologique : le départ, le trajet, le contrôle aux frontières, l'arrivée, les emplois, l'acculturation, les démêlés avec les autorités administratives, l'enfermement, les centres de rétention et l'expulsion.

Ainsi, parmi les diverses fictions portant à l'écran l'expérience migratoire, certaines mettent en images et en sons le départ et les passeurs (Frozen River, Hunt, 2008; Le Havre, Kaurismäki, 2011), les difficiles conditions du trajet, la faim et la promiscuité (The Immigrant, Chaplin, 1917; Utvandrama (Les émigrants), Troell, 1971; Ein Augenblick Freiheit [Pour un instant, la liberté], Riahi, 2008), les dangers physiques (Harragas, Allouache, 2010; La Pirogue, Touré, 2012), l'espoir et son cortège de rêves (El Norte [Au nord le paradis], Nava, 1983; Nuovomondo [La porte d'or], Crialese, 2006), les violences des polices frontalières (The Three Burials of Melquiades Estrada, Jones, 2005), les premiers pas du migrant au sein du pays d'accueil, son insertion physique et acoustique (Pane e cioccolata [Pain

\footnotetext{
${ }^{14}$ Voir le Festival cinéma et migrations d'Agadir.
} 
et chocolat], Brusati, 1974 ${ }^{15}$, The Piano, Campion, 1993; Stupeur et tremblements, Corneau, 2003; Eden à l'Ouest, Costa-Gavras, 2009), les violences subies (Sin nombre, Fukunaga, 2009; The Immigrant, Gray, 2013; Por Aqui Tudo Bem [Alda et Maria], Pascoal, 2011), les réactions de rejet, les épreuves humiliantes révélatrices de la suspicion en laquelle on les tient et les manquements qu'ils accusent à l'égard de la société de leur immigration (Angst essen Seele auf [Tous les autres s'appellent Ali], Fassbinder, 1974; Little Senegal, Bouchareb, 2001), l'isolement, l'épuisement (In America, Sheridan, 2002) puis les interactions avec leur communauté culturelle (Pane e cioccolata [Pain et chocolat], Brusati, 1974), l'acculturation et les éléments géopolitiques du pays d'origine (Va, vis et deviens, Mihaileanu, 2005; Persepolis, Paronnaud, Satrapi, 2007; Monsieur Lazhar, Falardeau, 2011), les divers emplois occupés (America America, Kazan, 1963; The Godfather: Part II, Coppola, 1974), l'exploitation des migrants (It's a Free World..., Loach, 2007), les rivalités entre immigrants et l'exploitation d'immigrants par d'autres immigrants (Biutiful, Iñárritu, 2010), l'engagement syndical (Bread and Roses, Loach, 2000), le commerce d'organes (Dirty Pretty Things, Frears, 2002), le conflit intergénérationnel (My Son the Fanatic, Prasad, 1997; L'ange de goudron, Chouinard, 2001), I'aide aux personnes en situation irrégulière (Welcome, Lioret, 2009; Samba, Nakache, Toledano, 2014), les démêlés avec les autorités administratives (Alambrista!, Young, 1977), l'enfermement et les centres de rétention (The Terminal, Spielberg, 2004; The Visitor, McCarthy, 2007; Illégal, Masset-Depasse, 2010; Né quelque part, Hamidi, 2012), la honte et la peur du retour au pays, la reconduite à la frontière et l'expulsion (Bled Number One, Ameur-Zaïmech, 2005).

Ces films offrent une compréhension sans complaisance ni condescendance de l'expérience migratoire et rendent compte de la manière dont les migrants intériorisent une certaine interprétation de la place qu'ils occupent dans la société d'accueil, dans les rapports de production et dans les relations de pouvoir qu'ils entretiennent avec les nationaux (notamment avec les employeurs).

Malgré la diversité de ces productions, l'immigration continue d'être, dans plusieurs d'entre elles (Pane e cioccolata [Pain et chocolat], El Norte [Au nord le paradis], It's a Free World..., Alambrista!, The Immigrant (2013), Eden à l'Ouest), une catégorie homogène et uniforme dont les membres sont réduits à une seule caractéristique, leur force de travail. Au sein de ces films, le migrant ne se conçoit que dans et par le travail et semble dénué de toute dimension culturelle, politique, affective, psychologique et psychique. Rares sont les cinéastes à mettre en images et en sons les impacts des violences, de l'isolement affectif, de la guerre, des persécutions, des menaces, des cauchemars, du stress d'acculturation ${ }^{16}$, de la peur et de l'emprisonnement sur la santé mentale de ces migrants ${ }^{17}$. Encore plus rares sont les exemples de comportements destructifs, de décompensation ${ }^{18}$, de prise en charge médicale. Être immigré et malade, c'est-à-dire requérant des soins thérapeutiques et cliniques, semble constituer alors un paradoxe à l'écran. Certaines de ces réalisations (Pane e cioccolata [Pain et chocolat], Eden à l'Ouest, Illégal, America America) se terminent en laissant le protagoniste principal isolé, exténué, hébété, en pleine situation de désorganisation culturelle et familiale et au seuil d'une perte du sens de la réalité. La maladie (les troubles psychiques, les pulsions d'autodestruction, etc.) et l'hospitalisation des migrants demeurent à ce jour peu traitées dans le cinéma fictionnel, alors que le septième art présente un grand intérêt pour la santé mentale des non-migrants et pour les intrigues se déroulant dans des espaces cliniques et thérapeutiques, comme nous l'avons vu précédemment (partie 2).

\footnotetext{
15 L'une des premières séquences de Pane e cioccolata [Pain et chocolat] (Brusati, 1974) est à cet égard très significative. Le protagoniste principal, Giovanni Garofalo, un immigré italien tout juste arrivé en Suisse, trouble l' " ordre acoustique » régnant dans un jardin public par la mastication de son sandwich. Brusati dresse un portrait saisissant de ces "personnes déplacées ", dépourvues de place (physique, affective, culturelle) appropriée dans l'espace environnemental et social de la société d'accueil. Ce film donne à comprendre le sens de cette non-place accordée au non-national.

${ }^{16}$ Le stress d'acculturation comprend les difficultés prémigratoires et post-migratoires. Selon Hopkins-Kavanagh et Sananikone (1981), il est plus important lorsque la « distance » entre la culture de l'immigrant et celle du groupe majoritaire est plus grande. II n'est pas forcément négatif et peut constituer une force positive propice au fonctionnement psychologique de la personne. (Sayegh et Lasry, 1993, p. 23-51).

${ }^{17}$ Déjà en 1952, Albert Béguin soulignait dans le numéro d’Esprit consacré à la « Misère de la psychiatrie »: « Progressivement, la marge des comportements tenus pour normaux s'est resserrée autour d'une notion d'utilité et de bien commun, qui tolère de moins en moins le mystère de l'être hors-série, improductif, de celui qui pèsent désormais de nouvelles malédictions " (p. 781).

18 Ces symptômes (stress d'acculturation, syndrome post-traumatique, etc.) sont souvent ceux que présentent les migrants (Beiser, Turner et Ganesan, 1989; Bernier, 1993; Dongier, Kiolet et Ledoux, 2007).
} 
Au vu de ces considérations sur les liens entre cinéma et maladie mentale, puis ceux entre cinéma et immigration, il apparaît que le corpus de films portant sur la maladie mentale au sein d'une expérience migratoire est faible (La faute à Voltaire, Kechiche, 2000). À notre connaissance, il n'existe pas d'autres longs métrages de fiction dont le protagoniste principal est un migrant malade. Ce constat impose de problématiser davantage la notion de migrant et de migration. Jusqu'à présent, le migrant a été envisagé comme une « personne déplacée dans l'espace et le temps, et neutralisée politiquement " (Sayad, 2006). En vue d'élargir notre corpus, cette acception de la migration gagne à être précisée en s'interrogeant sur la nature du déplacement, la durée et la direction de l'expérience migratoire ${ }^{19}$. Ainsi, toute migration peut être volontaire ou forcée, intérieure ou extérieure et temporaire ou permanente.

Ces trois nouveaux critères permettent de constituer un corpus d'analyse de films traitant de la santé mentale de personnes au sein d'un même pays ou entre plusieurs pays.

- Le recours au critère 1 permet d'élargir le corpus à tous les films abordant l'impact de l'esclavage (Amistad, Spielberg, 1997; Beloved, Demme, 1998; Planet of the Apes, Burton, 2001; Promised Land, Gitai, 2004; Vénus Noire, Kechiche, 2010; Rebelle, Nguyen, 2012) et des génocides sur la santé mentale de ces individus déplacés de manière non volontaire et forcée (réfugiés, demandeurs d'asile, exilés).

- Le critère 2 offre l'occasion d'examiner des films retraçant des mouvements intérieurs (exode rural, déplacement de personnes malades, etc.) au sein d'un même espace national (Scarecrow, Schatzberg, 1973; The Homesman, Jones, 2014) et d'apprécier les conséquences diégétiques de cette " migration intérieure» ${ }^{20}$ sur la santé mentale de ces personnes déplacées.

- Le critère 3 autorise l'examen d'une nouvelle catégorie fictionnelle. Il s'agit des films de guerre (notamment américains) mettant en lumière l'impact des conflits sur la santé mentale des soldats ayant séjourné temporairement en territoire étranger (et notamment le syndrome de stress post-traumatique ${ }^{21}$ ). Si une grande partie de ces films relate l'impossible retour de soldats américains dans leur communauté (Let There Be Light, Huston, 1946; Taxi Driver, Scorsese, 1976; Coming Home, Ashby, 1978; Rambo : First Blood, Kotcheff, 1982; Born on the Fourth of July, Stone, 1989; Jacob's Ladder, Lyne, 1990 ; In the Valley of Elah, Haggis, 2007; Jimmy P., Desplechin, 2013), certains dépeignent le processus de déshumanisation et les chocs émotionnels auxquels sont confrontés les soldats (Apocalypse Now, Coppola, 1979; Platoon, Stone, 1986; Full Metal Jacket, Kubrick, 1987), tandis que d'autres prennent comme protagonistes principaux des immigrants qui, par leur engagement militaire et leur sacrifice pour la patrie, deviennent pleinement américains (The Deer Hunter, Cimino, 1978). Cette catégorie fictionnelle centrée sur les soldats pourrait être élargie aux expatriés, aux étudiants et aux diplomates.

Ces critères internes au corpus nous semblent intéressants pour estimer non seulement la richesse et la diversité de ces productions, mais également pour considérer la manière dont elles sont susceptibles de mettre en images et en sons les multiples aspects communs et inhérents à l'expérience migratoire et à la maladie mentale. Effectuons à présent une brève incursion dans le cinéma documentaire en ayant à l'esprit les interrelations mentionnées cidessus entre immigration et santé mentale.

\footnotetext{
19 Mentionnons que d'autres facteurs tels que l'âge d'arrivée dans un nouveau pays, le genre, l'état civil, le niveau socioéconomique avant et après la migration, l'instruction, la profession et la possession d'un titre de séjour peuvent avoir un impact sur la santé mentale. Ces variables s'avèrent très pertinentes dans l'analyse de contenu filmique mais ne permettent pas un élargissement du corpus.

${ }^{20}$ La volonté est ici d'élargir la notion de migrant à des personnes déplacées qui n’entrent généralement pas dans le terme de migrants, car ne posant pas les mêmes problèmes d'ordre juridique (permis d'établissement, droit de travail, etc.).

21 Dans le cinéma américain, le Post-Traumatic Stress Disorder ou PTSD a déjà fait l'objet de diverses recherches (Kaes, 2010; Maseda et Dulin, 2012). Concernant le cinéma européen, voir Maseda (2011).
} 


\section{Incursion dans le cinéma documentaire}

À notre connaissance, le premier documentaire à mettre en lumière la santé mentale de migrants est Les Maîtres fous (1955) de Jean Rouch. Ce court-métrage tourné à Accra ${ }^{22}$ avec de jeunes travailleurs immigrés venus du Niger et du Mali donne à voir une Afrique en pleine transformation sociale, économique et politique. Rouch enregistre des cultes de possession nouveaux en réaction à la violence acculturatrice du colonialisme. Il est ainsi possible d'observer une réinterprétation du système colonial britannique dans le système de pensée des Haoukas. D'un point de vue ethnopsychiatrique, ces crises de possession participent d'un processus d'identification à l'agresseur où des Noirs intériorisent physiquement des situations de domination coloniale et se prennent pour des machines, des locomotives, des généraux, etc. Comme le rappelle Marc-Henri Piault, "possession, migration, aliénation coloniale sont les thèmes dominants de ce film et la perspective est résolument dynamique » $(2008$, p. 217). Si la migration de ces jeunes du Niger et du Mali vers les pays de la côte de l'Afrique occidentale, en particulier le Ghana et la Côte d'Ivoire, est volontaire, notons qu'elle est dans un premier temps extérieure (d'un pays à l'autre), puis intérieure. Ces cultes de possession se déroulent dans leur intégralité en brousse, à l'inverse du lieu de travail, la ville. Aucune mention dans le film ne précise la durée de ces migrations. Le documentaire se termine sur deux migrants en train de travailler devant le Mental Hospital et Rouch de conclure : "On ne peut s'empêcher de se demander si ces hommes d'Afrique ne connaissent pas certains remèdes qui leur permettent de ne pas être des anormaux, mais d'être parfaitement intégrés à leur milieu. Des remèdes que nous ne connaissons pas encore ${ }^{23}$.

Depuis les travaux de Rouch, quelques ethnocinéastes et certains documentaristes se sont intéressés à la santé mentale des migrants (notamment des réfugiés quittant leur pays pour sauver leur vie et présentant souvent de lourds traumatismes prémigratoires) et ont porté ce sujet à l'écran (J'ai rêvé d'une grande étendue d'eau, PetitJouvet, 2002; Sous la main de l'autre, Detours et Henry, 2011). Plus récemment, une série de trois courts-métrages d'animation intitulée Le parcours du demandeur d'asile (Le parcours géographique; Le parcours administratif; L'accès au soin en psychiatrie) a été réalisée en France en 2012 au sein du Réseau Samdarra (Santé mentale, précarité, demandeur d'asile et réfugiés en Rhône-Alpes) par Marina Roel, Etienne Guiol et Melissa Plantaz. Elle a pour public cible les professionnels et les bénévoles en contact avec les demandeurs d'asile ${ }^{24}$. La visée est ouvertement pédagogique et a pour but de sensibiliser à la compréhension des problèmes des migrants et de clarifier la complexité les diverses étapes auxquelles sont confrontés les migrants dans leur demande d'asile et dans leur prise en charge, principalement en France. Le style graphique est épuré à dessein et l'animation est minimaliste, en vue de rendre le message le plus efficace et universel possible; le ton du commentaire est neutre. Très factuels et informatifs, ces films ne versent dans aucun pathos.

Dans le cadre de cet article, deux de ces films ont retenu notre attention : Le parcours géographique et L'accès au soin en psychiatrie.

\section{Le parcours géographique}

Le premier dresse le portrait de trois hommes qui, dans la file d'attente de la préfecture, viennent demander l'asile. D'emblée une prise de recul spatiale et temporelle permet de saisir la singularité et le parcours de chacun. Monsieur A vient de la République démocratique du Congo (un pays en guerre) et a été recruté comme enfant soldat à l'âge de 12 ans. Brutalisé, drogué, violé, il est contraint de commettre des atrocités. Au bout de 4 ans, il réussira à s'enfuir. Monsieur B, lui, vient du Kosovo, il est accusé d'avoir pendant la guerre aidé des amis d'une autre ethnie. Menaces, chantage, violence l'obligent à quitter son pays. Monsieur C, Sri-Lankais, est cadre d'un parti politique minoritaire. En proie à des mesures d'intimidation, il décide de partir avec sa femme et deux de ses enfants. La volonté est ici de dépeindre le vécu des demandeurs d'asile (violence exacerbée, précarité, insécurité,

\footnotetext{
${ }^{22}$ Capitale de la Gold Coast qui allait devenir la première colonie africaine à gagner son indépendance sous le nom de Ghana.

${ }^{23}$ Remarquons que Roger Bastide partage une posture similaire à celle de Rouch dans Le Rêve, la Transe et la Folie : " nous, civilisés, avons rompu avec ces canaux de communication en naturalisant nos rêves, en enroulant nos malades mentaux dans des camisoles chimiques pour en faire des imitations de normaux, bref en rejetant le surnaturel pour le traduire en d'autres langues, plus satisfaisantes pour notre raison cartésienne, biologique et sociologique » $(1972$, p. 5).

${ }^{24}$ Les trois films d'animation sont disponibles en accès gratuit sur le site du Réseau, à l'adresse : http://www.samdarra.fr
} 
séparation familiale, etc.) et de sensibiliser aux conséquences de ce vécu sur la santé mentale de ces personnes. Ici, la migration est forcée, extérieure et permanente.

L'un des intérêts de ce film est d'associer dans l'esprit du spectateur à chaque migrant une histoire singulière, par un retour sur le temps et l'espace antérieurs à l'émigration, alors que, comme le rappelle Schütz, " du point de vue du nouveau groupe, l'étranger est toujours un homme sans histoire » (2003, p. 20).

\section{L'accès au soin en psychiatrie}

Le second film dresse le portrait de Monsieur $\mathrm{P}$, arrivé en France il y a deux mois et qui, depuis quelque temps, se sent très mal et craint de devenir fou. En quelques minutes nous sont présentées les multiples difficultés rencontrées par un migrant (qui n'est pas passé l'acte ou n'a pas décompensé) pour avoir accès aux soins psychiatriques en France. Ce sont les difficultés communicationnelles liées à la barrière de la langue, le manque d'informations sur la situation et la couverture médicale du patient, les souvenirs douloureux des interrogatoires lorsque le migrant se trouve dans le bureau du médecin, l'importance d'un interprète professionnel, le besoin d'un relais associatif pour le soutien aux professionnels, le manque de relais d'information entre diverses équipes, la difficulté enfin à déterminer si le migrant nécessite un accompagnement social ou médical.

Cette approche non personnalisée présente dans ces documentaires met en lumière la difficile expression du malêtre des migrants, à quel point cette incommunicabilité entrave l'aide du personnel médical et la nécessaire multiplicité des rencontres afin d'établir une relation de confiance. Derrière des images impersonnelles se cachent des expériences sociales très concrètes et complexes. Ainsi, cette série permet de souligner comment la condition de migrant est susceptible de générer des situations problématiques et vulnérabilisantes. Le migrant victime de violences doit non seulement, comme tout étranger, " remettre en question à peu près tout ce qui semble aller de soi aux membres du groupe qu'il aborde » (Schütz, 2003, p. 19), mais également lutter contre un vécu douloureux et omniprésent (visions-souvenirs) qui altère sa santé et son quotidien dans le pays d'accueil.

\section{Conclusions}

Au sortir de ce parcours réflexif, il apparaît d'une part que nombre de cinéastes n'hésitent pas à porter à l'écran la maladie mentale et, d'autre part, que la figure de l'étranger est présente de manière significative dans plusieurs cinématographies nationales ${ }^{25}$. En revanche, le cinéma de fiction aborde rarement la santé mentale des migrants. En effet, les perceptions collectives ne semblent que privilégier la force physique du migrant au détriment de son bien-être, faisant souvent fi de l'impact de son vécu prémigratoire (violences, menaces, séparation familiale, etc.) et de sa situation actuelle (morcellement culturel, dépatriement, déclassement social, etc.) sur sa santé mentale. Migrants et malades semblent être un inconciliable au grand écran. Il semble peu aisé de représenter une double exclusion-stigmatisation : le migrant souffrant de troubles psychiques est doublement hors normes, notamment avec les mesures de criminalisation et de psychiatrisation actuelles. Par conséquent, cet article n'a pas permis de répondre à la série d'interrogations initiales relatives au mode de figuration de la santé mentale en contexte migratoire. En l'absence d'un corpus de films sur cette thématique, nous n'avons pu montrer que le cinéma fictionnel était source de stéréotypie ou de rupture avec des représentations réductrices, ni analyser la mise en images et en sons des impacts des violences, de l'isolement affectif, de la guerre, des persécutions, des menaces et des troubles du sommeil sur la santé mentale des migrants.

Ces interrogations demeurent heuristiques - comme nous avons pu le voir dans le cas du cinéma documentaire d'animation à visée pédagogique, dont le but est de sensibiliser à la compréhension des problèmes des migrants et de clarifier la complexité les diverses étapes auxquelles sont confrontés les migrants dans leur demande d'asile et dans leur prise en charge principalement en France - et gagnent à être traitées dans des analyses futures en élaborant de nouveaux corpus de films de fiction. Une problématisation de la notion de migrant permet de pallier cette aporie en élaborant trois nouveaux critères de constitution du corpus filmique, proposant ainsi des pistes de recherche quant à une meilleure connaissance des représentations des migrants et des pratiques réelles (politiques

\footnotetext{
${ }^{25}$ Voir la revue Décadrages, n 14, 2009, consacrée à "Cinéma et migration 》, ainsi que CinémAction, n 8, été 1979, "Cinémas de l'émigration » et CinémAction, n²4, janvier 1983, "Cinémas de l'émigration 3 ».
} 
d'accueil en matière de santé des migrants, acteurs et dispositifs cliniques, mutations professionnelles contemporaines).

\section{Bibliographie}

Agier, M. (2013). La condition cosmopolite. L'anthropologie à l'épreuve du piège identitaire. Paris : La Découverte.

Bastide, R. (1965). Sociologie des maladies mentales. Paris : Flammarion.

Bastide, R. (1972). Le Rêve, la Transe et la Folie. Paris : Flammarion.

Becker, H. (2009). Comment parler de la société : artistes, écrivains, chercheurs et représentations sociales. Paris : La Découverte.

Béguin, A. (1952). Qui est fou? Esprit, 197, 777-788.

Beiser, M., Turner, R. et Ganesan, S. (1989). Catastrophic Stress and Factors Affecting Its Consequences among Southeast Asian Refugees. Social Science and Medicine, 28(3), 183-195.

Benbassa, E. (2007). La souffrance comme identité. Paris : Fard.

Ben Jelloun, T. (1977). La plus haute des solitudes. Paris : Seuil.

Bernier, D. (1993). Le stress des réfugiés et ses implications pour la pratique et la formation. Service social, 42(1), 81-99.

Bovier, F. (2011). Regards sur l'« impouvoir » : le « cinéma direct » de Ruspoli, de la terre à l'asile. Décadrages, 18, 14-31.

Calderon, P. (2003). Michel Foucault par lui-même [Vidéo]. Paris : Arte France, Paris : BFC Productions.

CinémAction n 8, été 1979, "Cinémas de l'émigration ».

CinémAction $n^{\circ} 24$, janvier 1983, "Cinémas de l'émigration 3 ».

Décadrages n 14, 2009, « Cinéma et migration ».

Devereux, G. (1977). Essais d'ethnopsychiatrie générale. Paris : Gallimard.

Devereux, G. (1998). Psychothérapie d'un Indien des Plaines. Paris : Fayard.

Dongier, P., Kiolet, M. et Ledoux, I. (2007). La santé mentale des immigrants. Le Médecin du Québec, 42(3), 33-39.

Ey, H. (1952). Anthropologie du malade mental. Esprit, 197, 891-896.

Fassin, D. et Rechtman, R. (2011). L'empire du traumatisme. Enquête sur la condition de victime. Paris : Flammarion.

Foucault, M. (2005). Naissance de la clinique. Paris : Presses universitaires de France.

Freud, S. (1985). L'inquiétante étrangeté et autres essais. Paris : Gallimard. (1 ${ }^{\mathrm{e}}$ édition française, 1933)

Gordon, R. B. (2013). De Charcot à Charlot : mises en scène du corps pathologique. Rennes : Presses universitaires de Rennes.

Hamon-Sirejols, C. et Gardies, A. (dir.). (1997). Le Spectaculaire. Lyon : Aléas.

Hopkins-Kavanagh, K. et Sananikone, P. (1981) Migration, mental health and the Laotian refugee. Migration News, 1, 15-23.

Kaes, A. (2010). Shell Shock Cinema: Weimar Culture and the Wounds of War. Princeton, NJ : Princeton University Press.

Linton, R. (1968). De l'homme. Paris : Minuit. 
Lipiansky, E.-M., Taboada-Leonetti, I. et Vasquez, A. (1990). Introduction à la problématique de l'identité. Dans C. Camilleri, J. Kastersztein, E.-M. Lipiansky, H. Malewska-Peyre, I. Taboada-Leonetti et A. Vasquez (dir.), Stratégies identitaires (p. 7-26). Paris : Presses universitaires de France.

Maseda, R. (2011). The Secret Lives of Women in the Aftermath of Combat: Female Post Traumatic Stress Disorder in European Cinema. The International Journal of the Humanities, 9(9), 123-134.

Maseda, R. et Dulin, P. L. (2012). From Weaklings to Wounded Warriors: The Changing Portrayal of War-related Post Traumatic Stress Disorder in American Cinema. 49th Parallel, 30 (en ligne). Téléchargeable à partir de la page : http://49thparalleljournal.org/category/2012/issue-30/

Moscovici, S. (1991). Psychologie des minorités actives. Paris : Les Presses Universitaires de France.

Morin, E. (1956). Le cinéma ou l'homme imaginaire. Essai d'anthropologie. Paris : Minuit.

Morin, E. (2009). L'esprit du temps. Paris : Armand Colin/INA. (1 édition, 1962)

Parsons, T. (1955). Éléments pour une sociologie de l'action. Paris : Plon.

Piault, M.-H. (2008). Anthropologie et cinéma. Paris : Téraèdre.

Quemada, B. (dir.). (1992). Trésor de la langue française (vol. 15). Paris : Gallimard.

Ricœur, P. (1995). Le juste. Paris : Esprit.

Saïd, E. (2008). Réflexions sur l'exil. Paris : Actes Sud.

Sayad, A. (2006). L'immigration ou les paradoxes de l'altérité (tome 1. L'illusion du provisoire). Paris : Raisons d'agir.

Sayegh, L. et Lasry, J.-C. (1993). Acculturation, stress et santé mentale chez des immigrants libanais à Montréal. Santé mentale au Québec, 18(1), 23-51.

Schütz, A. (2003). L'Étranger, suivi de L'Homme qui rentre au pays. Paris : Allia.

Simmel, G. (2010). Sociologie. Études sur les formes de la socialisation. Paris : Presses universitaires de France.

Sontag, S. (2005). La maladie comme métaphore. Paris : Bourgeois.

Sorlin, P. (1972). Cinéma et société. Études, 336(1), 121-129.

Sorlin, P. (1977). Sociologie du cinéma : ouverture pour l'histoire de demain. Paris : Aubier Montaigne.

Vasquez, A. (1990). Les mécanismes des stratégies identitaires : une perspective diachronique. Dans C. Camilleri, J. Kastersztein, E.-M. Lipiansky, H. Malewska-Peyre, I. Taboada-Leonetti et A. Vasquez (dir.), Stratégies identitaires (p. 143-171). Paris : Presses universitaires de France.

Zarifian, É. (2006). La psychiatrie et le cinéma : une image en miroir. Les Tribunes de la santé, 11, 39-45.

\section{Filmographie}

$37^{\circ} 2$ le matin (France)

A Beautiful Mind (États-Unis)

A Dangerous Method (Royaume-Uni / Allemagne / Canada / Suisse)

A Woman Under the Influence (États-Unis)

Alambrista! (États-Unis)

America America (États-Unis)

Amistad (États-Unis)

An Angel at My Table (Nouvelle-Zélande / Australie / Royaume-Uni)

Angst essen Seele auf [Tous les autres s'appellent Ali] (Allemagne de l'Ouest)

Apocalypse Now (États-Unis)

Beloved (États-Unis)

Birdy (États-Unis)

Biutiful (Mexique / Espagne)

Alterstice - Revue Internationale de la Recherche Interculturelle, vol. 4, $n^{\circ} 2$ 


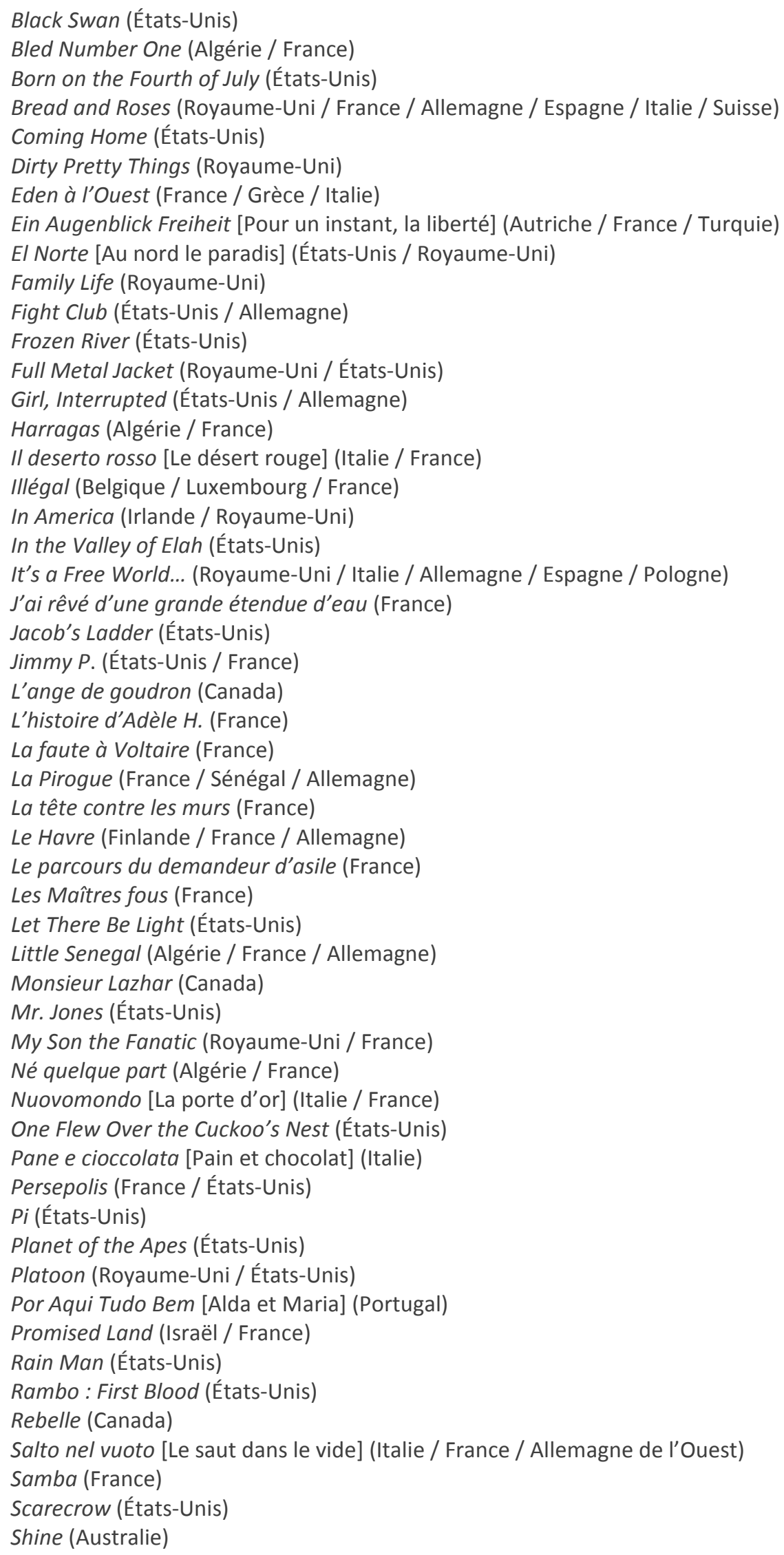




\author{
Shock Corridor (États-Unis) \\ Shutter Island (États-Unis) \\ Sin nombre (Mexique / États-Unis) \\ Sous la main de l'autre (France / Suisse / Belgique) \\ Stupeur et tremblements (France / Japon) \\ Suddenly, Last Summer (États-Unis) \\ Taxi Driver (États-Unis) \\ The Deer Hunter (Royaume-Uni / États-Unis) \\ The Godfather : Part II (États-Unis) \\ The Homesman (France / États-Unis) \\ The Immigrant (1917) (États-Unis) \\ The Immigrant (2013) (États-Unis) \\ The Madness of King George (Royaume-Uni) \\ The Mosquito Coast (États-Unis) \\ The Piano (Nouvelle-Zélande / Australie / France) \\ The Terminal (États-Unis) \\ The Three Burials of Melquiades Estrada (France / États-Unis) \\ The Visitor (États-Unis) \\ Utvandrama (Les émigrants) (Suède) \\ $V a$, vis et deviens (France / Israël / Belgique / Italie) \\ Vénus Noire (France / Belgique) \\ Welcome (France)
}

